\title{
A vagina-escola: \\ seminário interdisciplinar sobre violência contra a mulher \\ no ensino das profissões de saúde
}

\author{
Carmen Simone Grilo Diniz ${ }^{(a)}$ \\ Denise Yoshie Niy ${ }^{(b)}$ \\ Halana Faria de Aguiar Andrezzo ${ }^{(\mathrm{c})}$ \\ Priscila Cavalcanti Albuquerque Carvalho(d) \\ Heloisa de Oliveira Salgado(e)
}

\section{Antecedentes}

Desde a década de 1950, quando se criaram as primeiras associações a apontar a "crueldade contra as grávidas", numerosas iniciativas, grupos e redes se formaram com a finalidade de visibilizar e reduzir as várias formas de desrespeito e abuso contra as mulheres na assistência ao parto ${ }^{1}$. O que no Brasil, hoje, chamamos de "violência obstétrica" é um tema antigo, que eclode periodicamente, sob diferentes termos².

Como profissionais de saúde, somos socializados a crer que nosso atendimento é sempre uma ajuda às mulheres, e ficamos chocados, às vezes hostis, diante das narrativas das parturientes que percebem nossa assistência como um abuso, um desrespeito, uma forma de indignidade ${ }^{2}$. As mulheres, por sua vez, receiam falar sobre o assunto, temendo melindrar aqueles dos quais podem um dia depender. É como se falar do problema provocasse sua existência, de forma que, muitas vezes, se prefere o silêncio, ou a formulação de narrativas menos ameaçadoras às relações sociais. No Brasil, a Rede pela Humanização do Parto e do Nascimento (Rehuna), em sua carta de fundação (1993), parte do reconhecimento das "circunstâncias de violência e constrangimento em que se dá a assistência" 3 . Porém, a organização, deliberadamente, abriu mão de falar do enfrentamento à violência, ao privilegiar termos como 'humanizar o parto', 'promover os direitos humanos das mulheres' e 'melhorar o acolhimento', temendo uma reação hostil dos profissionais diante da acusação de violência'.

Pode-se dizer que a medicina baseada em evidências (MBE), que começa na área de saúde perinatal ${ }^{5}$, é um movimento de profissionais de saúde aliados a movimentos de mulheres ${ }^{6}$ preocupado em dar visibilidade às rotinas de sofrimento desnecessário no parto e aos seus efeitos prejudiciais, como: proibição da presença de familiares, imobilização física, privação de água e alimentos, lavagens retais, raspagem de pelos pubianos, entre outras. Este movimento chama a

(a) Departamento de Saúde Materno-infantil, Faculdade de Saúde Pública, Universidade de São Paulo (USP). Av. Dr. Arnaldo, 715 sala 203. São Paulo, SP, Brasil. 01246-904. sidiniz@usp.br (b,e) Doutoranda em Ciências, Faculdade de Saúde Pública, USP. denise.niy@gmail. com; hellosalgado@ gmail.com (c,d) Mestranda em Ciências, Faculdade de Saúde Pública, USP. halanitaflor@ gmail.com; pcacadvogada@ gmail.com 
atenção para intervenções agressivas praticadas rotineiramente, como episiotomia (corte da vagina durante o parto), fórceps, aceleração do parto, entre outras ${ }^{7}$. Nas últimas três décadas, o movimento da MBE construiu a evidência 'dura' de ensaios clínicos e revisões sistemáticas a favor de rotinas menos agressivas, mais amigáveis a mulheres e bebês, protegendo-os de abusos. Destacam-se os benefícios: da atenção ao conforto físico e emocional da mulher, da presença de acompanhantes e doulas ${ }^{8,9}$, da liberdade de movimentar-se e escolher a posição de parir, da valorização da integridade genital materna, do contato pele a pele entre mãe e bebê na primeira hora de vida, do corte tardio do cordão, entre outros ${ }^{10}$.

Em países permeáveis às evidências científicas, este movimento foi muito efetivo em questionar a tradição, e em mudar as rotinas de assistência e os valores subjacentes ${ }^{10}$. Porém, em outros contextos, sobretudo nos países de renda média com uma medicina pouco regulada, a prática clínica tem sido pouco permeável às evidências, como no caso do Brasil ${ }^{11}$, criando uma contradição adicional: a permanência, não regulada, de intervenções reconhecidamente inseguras e dolorosas, o que configura uma forma de violação de direitos 'nova', sendo a novidade o seu reconhecimento como problema e não sua ocorrência.

Para os movimentos sociais que se organizam pela mudança do modelo de atenção ao parto, a permanência de uma assistência agressiva tem como finalidade coagir à cesárea, tornada assim, comparativamente, uma alternativa menos aflitiva, dolorosa e insegura. Segundo estes movimentos, "chega de parto violento para vender cesárea"12.

\section{Novas narrativas sobre direitos e sobre abusos}

No Brasil, as seguidas tentativas de políticas públicas dirigidas à mudança da assistência ao parto tiveram efeito limitado, com taxas altas e estagnadas de morbimortalidade materna e aumento constante das cesáreas e da prematuridade. As instituições reguladoras resistem a exercer seu papel, e, desse modo, não favorecem a efetivação das políticas. Ao mesmo tempo, o aparelho formador continua a reproduzir práticas instituídas há décadas, desconsiderando os conhecimentos científicos acumulados mais recentemente, e abordando a mulher não como sujeito de direitos, mas como objeto de sua atuação ${ }^{13,14}$. Diante disso, os movimentos sociais têm apelado a recursos como o Ministério Público, buscando coibir abusos e regular minimamente as intervenções no parto. Isso tem resultado em inúmeras audiências públicas sobre abuso de intervenções e violência obstétrica em todo o país.

Estas audiências focam questões como o abuso de cesárea no setor suplementar e temas diversos relativos à violência obstétrica. No primeiro caso, uma ação civil pública foi iniciada ${ }^{15}$ diante de denúncia da Rede Parto do Princípio (2006) ${ }^{16}$, resultando na edição de normativa pela Agência Nacional de Saúde Suplementar, em 2015, com vistas a regular a realização de cesarianas ${ }^{17}$. Ainda que a efetividade de tal norma seja questionável, é inegável que uma de suas componentes - a obrigatoriedade de os convênios divulgarem as taxas de cesarianas dos profissionais de saúde e maternidades - afeta o equilíbrio de poderes ora estabelecido.

Nestas audiências, movimentos sociais argumentam que a permanência do ensino acrítico de procedimentos dolorosos e, por vezes, desnecessários reflete o ensino de valores que atribuem predominância aos profissionais de saúde, ao mesmo tempo em que despersonificam as mulheres ${ }^{18-23}$. Assim, por exemplo, na prática, ensina-se, aos futuros profissionais, que a paciente não tem direito à escolha ou à recusa informada, e que as necessidades de ensino dos treinandos são mais importantes que a autonomia ou a integridade corporal das parturientes. Nesse sentido, foram consideradas violações de direitos: o uso excessivo ('liberal') de procedimentos sem base em evidências, potencialmente danosos, sem problematizar os efeitos adversos em mães e bebês; bem como as intervenções feitas sem autorização da paciente, e, mesmo, à revelia de sua desautorização expressada verbalmente e por escrito, apenas com a finalidade de treinar técnicas cirúrgicas (como episiotomias e fórceps 'didáticos').

No segundo semestre de 2014, em duas dessas audiências em São Paulo ${ }^{21,23}$, um depoimento foi especialmente perturbador: Mary Dias, estudante universitária, negra, relatou que, em um hospitalescola, recebeu duas episiotomias em um mesmo parto. Ela conta que ouviu um dos profissionais falar 
para dois alunos: "você corta à direita e o outro corta à esquerda", supostamente para que ambos tivessem a oportunidade de treinar o corte e a sutura em sua vagina.

O ensino de obstetrícia no Brasil, tradicionalmente, requer que o aluno realize um certo número de procedimentos para que seja avaliado. Em outros países, a episiotomia e o fórceps, por exemplo, são treinados, preferencialmente, em modelos sintéticos e peças específicas, ao passo que, no Brasil, muitos profissionais relatam começar seu treinamento das habilidades cirúrgicas nas pacientes, via de regra usuárias do SUS. Vários autores mostram a cultura disseminada do ensino do uso não informado, não consentido, das vaginas das parturientes pobres pelos alunos de medicina e outras profissões, para fins de treinamento de habilidades ${ }^{24-27}$. Tais abusos permanecem normalizados por uma cultura institucional que não os reconhece como violações de direitos $12,24,27$.

O ensino da técnica cirúrgica não deveria estar descolado do ensino de valores e da ética profissional, e o caso de Mary Dias reflete a inter-relação destas duas dimensões do ensino: por um lado, a resistência ao reconhecimento das evidências científicas que recomendam o uso da episiotomia somente em situações muito excepcionais ${ }^{28}$ ou, mesmo, sua abolição ${ }^{29}$; e, por outro, a resistência ao reconhecimento e ao ensino dos direitos humanos das pacientes, bem como de seu direito à escolha e à recusa informada. Por fim, resta salientar que a situação expressa o desconhecimento do direito da mulher à integridade corporal, que deve ser preservada sempre que possível ${ }^{2,12}$.

\section{O seminário "A vagina-escola"}

A fim de promover o diálogo entre os setores envolvidos, em março de 2015, realizamos, na Faculdade de Saúde Pública da USP, um evento intitulado "A vagina-escola: seminário sobre violência contra a mulher no ensino das profissões de saúde", como parte da disciplina eletiva de graduação "Gênero, raça/etnia, sexualidades e saúde pública". O termo "vagina-escola" foi usado por entrevistados em uma pesquisa do grupo ${ }^{4}$, comentando a formação dos profissionais: seria relacionado ao fato de que, em muitas escolas médicas, a episiotomia é a primeira oportunidade dos médicos, de qualquer especialidade, de praticar habilidades cirúrgicas, cortando e suturando a vagina das mulheres pobres.

O seminário ocorreu em meio aos protestos contra a impunidade de casos de estupro e assédio sexual na USP, em especial, na Faculdade de Medicina, e que chegaram a motivar uma CPI na Assembleia Legislativa ${ }^{30}$. Sabemos que tais problemas de violência contra a mulher, em seu sentido mais amplo, não são exclusivos de uma faculdade ou de uma universidade. Porém, algumas instituições têm sido mais lentas no seu enfrentamento, o que reforça a importância de pressões externas para superar a inércia institucional.

As formas de violência mencionadas - por um lado, o abuso sexual, sua invisibilidade e impunidade, e por outro, a realização não consentida de intervenções sobre os genitais no parto, para além de suas indicações clínicas e apenas com finalidades didáticas - são faces do mesmo fenômeno. Refletem uma sociedade na qual persistem a normalização e a impunidade do acesso abusivo ao corpo feminino, reproduzindo hierarquias sociais de gênero, classe e raça/etnia.

A introdução do seminário foi uma reflexão sobre como as relações de gênero e demais marcadores sociais da diferença são estruturantes da assistência ao parto e dos abusos identificados. O depoimento de Mary Dias ao Ministério Público Federal foi reproduzido e, em seguida, os convidados comentaram o caso a partir de diferentes perspectivas. O professor Ivan França Junior falou sobre como ensinar direitos humanos, ao passo que a procuradora Ana Previtalli descreveu as ações do Ministério Público Federal sobre episiotomia e violência obstétrica. A importância e os mecanismos de regulação das práticas de saúde e de seus abusos constituíram tema da professora Marília Louvison, enquanto o professor Luís Eduardo Batista, do Instituto de Saúde, falou sobre racismo institucional no parto. Por fim, a professora Roselane Gonçalves, da Escola de Artes, Ciências e Humanidades, abordou as alternativas no ensino da assistência ao parto e da proteção do períneo. Os colegas implicados na narrativa de Mary Dias também foram convidados para o seminário, mas preferiram esperar o resultado da sindicância que foi aberta para apurar a denúncia.

Discutiu-se que as pessoas aderem a normas jurídicas por dois mecanismos: por estarem racionalmente convencidas de que a norma é justa, ou por coerção externa. Por essa razão, defendeu- 
se que, paralelamente a processos educativos, deve haver a correta aplicação do direito, pondo fim à invisibilidade e à impunidade dos casos de violência contra a mulher. Foi discutida a importância de desnaturalizar a concepção de que os profissionais de saúde têm o direito de acessar livremente o corpo das mulheres, sem seu consentimento informado, não apenas nos treinamentos em episiotomia, como, também, em toques vaginais repetidos, descolamentos de membranas, manobras de kristeller e, mesmo, nas indicações de cesárea.

Estas ações inovadoras são voltadas para visibilizar, prevenir e remediar esta forma de violência nas práticas de saúde, no âmbito público, privado, e na formação de recursos humanos; bem como para incentivar os governos e as instituições parceiras para pesquisas e ações, incluindo:

1) a incorporação e o ensino de evidências científicas sobre as intervenções no parto, inclusive, a promoção da integridade genital das mulheres;

2) o ensino da relação médico-paciente e dos direitos das mulheres, incluindo a proteção e promoção do direito à autonomia e à escolha informada;

3) o fim do uso desregulado e sem indicações médicas dos corpos das pacientes como material de ensino ('procedimentos didáticos'), com a remodelagem do ensino prático de intervenções, inclusive, cirúrgicas;

4) a identificação e responsabilização (accountability) dos 'abusos consensuais' (quando há um consenso entre os envolvidos de que se trata de um abuso), como fazer duas episiotomias na paciente apenas com fins didáticos;

5) a regulação de práticas médicas por meio da publicização de informações a esse respeito (como dos procedimentos realizados na assistência ao parto), a incorporação de protocolos e auditorias clínicas, com o apoio necessário das gestões locais.

O seminário veio em momento especialmente oportuno, acompanhando a recente declaração da OMS intitulada "Prevenção e eliminação de abusos, desrespeito e maus-tratos durante o parto em instituições de saúde" ${ }^{31}$. Este reconhecimento internacional reforça sua importância como tema global, e a urgência de intervenções para seu controle e prevenção.

\section{Colaboradores}

As autoras participaram, igualmente, da organização do evento que deu origem ao artigo, da redação do artigo e da revisão do texto final.

\section{Referências}

1. Goer H. Cruelty in maternity wards: fifty years later. J Perinat Educ [Internet]. 2010 [cited 2015 Sept 9]; 19(3):33-42. Available from: http://www.pubmedcentral.nih.gov/ articlerender.fcgi? artid $=2920649 \&$ tool $=$ pmcentrez\&rendertype $=$ abstract.

2. Tesser CD, Knobel R, Andrezzo HFA, Diniz SG. Violência obstétrica e prevenção quaternária: o que é e o que fazer. Rev Bras Med Fam Com [Internet]. 2015 [cited 2015 Sept 9]; 10(35):1. Available from: http://www.rbmfc.org.br/rbmfc/article/view/1013. 
3. ReHuNA - Carta de Campinas. In: Rattner D, Trench B, organizadores. Humanizando nascimentos e partos. São Paulo: Editora Senac; 2005. p. 171-5.

4. Diniz CSG. Humanização da assistência ao parto no Brasil: os muitos sentidos de um movimento. Cienc Saude Colet. 2005; 10(3):627-37. doi:10.1590/S141381232005000300019 .

5. Jaeschke R, Guyatt GH. What is evidence-based medicine? Semin Med Pract. 1999; 2(3):3-7.

6. Graham I. Episiotomy: challenging obstetric interventions. Oxford: Blackwell Science; 1997.

7. WHO. Appropriate technology for birth. Lancet. 1985; 326(8452):436-7. doi:10.1016/ S0140-6736(85)92750-3.

8. Hodnett E, Gates S, Hofmeyr G, Sakala C. Continuous support for women during chidlbirth. Cochrane Database Syst Rev. 2012; 1(10). doi:10.1002/14651858.CD003766. pub3.

9. Hodnett ED. Pain and women's satisfaction with the experience of childbirth: a systematic review. Am J Obstet Gynecol [Internet]. 2002 [cited 2015 Aug 18]; 186 Supl 5:S160-S172. Available from: http://www.ncbi.nlm.nih.gov/pubmed/12011880

10. Enkin M, Keirse MJNC, Neilson J, Crowther C, Duley Leila, Hodnett E. et al. Guia para atenção efetiva na gravidez e no parto. 3a ed. Rio de Janeiro: Guanabara Koogan; 2005.

11. Leal MC, Pereira APE, Domingues RMSM, Rosa Maria Soares Madeira, Theme Filha $M M$, Dias $M A B$, Nakamura-Pereira $M$, et al. Intervenções obstétricas durante o trabalho de parto e parto em mulheres brasileiras de risco habitual. Cad Saude Publica. 2014; 30 Supl. 1:S17-S32. doi:10.1590/0102-311X00151513.

12. Diniz SG. O renascimento do parto, e o que o SUS tem a ver com isso. Interface (Botucatu) [Internet]. 2014 [acesso 2015 Out 14]; 18(48):217-20. Disponível em: http://www.scielo.br/scielo.php?script=sci_arttext\&pid=S141432832014000100217\&lng=en. http://dx.doi.org/10.1590/1807-57622013.0910.

13. Hotimsky SN, Schraiber LB. Humanização no contexto da formação em obstetrícia. Cienc Saude Colet. 2005; 10(3):639-49.

14. Hotimsky SN. A formação em obstetrícia: competência e cuidado na atenção ao parto [tese]. São Paulo (SP): Faculdade de Medicina da Universidade de São Paulo; 2007.

15. Ministério Público Federal. Ação Civil Pública n. 0017488-30.2010.4.03.6100. Dispõe sobre a proteção dos direitos dos consumidores usuários de planos de saúde privados a obterem adequada informação e prestação de serviços médicos obstétricos. São Paulo: MPF-SP; 2010 [acesso 2015 Out 13]. Disponível em: http://www.prsp.mpf.gov.br/salade-imprensa/pdfs-das-noticias/Inicial\% 20-\% 200017488-30.2010.4.03.6100_cesarianas. pdf

16. Parto do Princípio. Representação n. 1.34.001.004458/2006-98. Sobre o abuso das cesarianas, ocorridas no Brasil, na rede privada de saúde, causando prejuízo a milhares de mulheres e crianças, requerendo providências urgentes contra essa situação. São Paulo; 2006 [acesso 2015 Out 13]. Disponível: http://media.wix.com/ ugd/2a51ae_0cda7bfcd82f4ae0a2aa1e04e6cb631b.pdf

17. Agência Nacional de Saúde Suplementar. Resolução Normativa n. 368/2015. Dispõe sobre o direito de acesso à informação das beneficiárias aos percentuais de cirurgias cesáreas e de partos normais, por operadora, por estabelecimento de saúde e por médico e sobre a utilização do partograma, do cartão da gestante e da carta de informação à gestante no âmbito da saúde suplementar [Internet]. Rio de Janeiro: ANS; 2015 [acesso 2015 Out 13]. Disponível em: http://www.ans.gov.br/index2.php?option=com legislacao\&view $=$ legislacao\&task $=$ TextoLei\&format $=$ raw $\& i d=2892$ 
18. Niy DY, Silva DRAD. Hospitais de ensino em obstetrícia: campo de violação dos direitos das mulheres. In: Direitos Humanos no Brasil 2015. São Paulo: Rede Social de Justiça e Direitos Humanos. (no prelo).

19. Freitas H. Ministério faz audiência pública no dia 3 para debater violência no parto [Internet]. Campo Grande News [acesso 2015 Set 1]. Disponível em: http://www. campograndenews.com.br/cidades/interior/ministerio-faz-audiencia-publica-no-dia-3para-debater-violencia-no-parto.

20. Ministério Público Federal. Parto do Princípio e Ministério Público Federal debatem violência obstétrica com movimentos sociais de mulheres [Internet]. 2014 [acesso 2009 Maio 20]:1-2. Disponível em: http://www.prpa.mpf.mp.br/news/2014/parto-doprincipio-e-ministerio-publico-federal-debatem-violencia-obstetrica-com-movimentossociais-de-mulheres

21. Ministério Público Federal. MPF em São Paulo realiza audiência pública para debater episiotomia e humanização do nascimento [Internet]. 2014 [acesso 2015 Set 1]. Disponível em: http://www.prsp.mpf.mp.br/sala-de-imprensa/noticias_prsp/16-10-14-2013-mpfem-sao-paulo-realiza-audiencia-publica-para-debater-episiotomia-e-humanizacao-donascimento

22. Ministério Público Federal. MPF/RS e MP/RS realizarão audiência pública sobre humanização da assistência ao parto [Internet]. 2014 [acesso 2015 Out 13]. Disponível em: http://noticias.pgr.mpf.mp.br/noticias/noticias-do-site/copy_of_geral/mpf-rs-e-mprs-realizarao-audiencia-publica-sobre-humanizacao-da-assistencia-ao-parto.

23. Ministério Público do Estado de São Paulo. Violência obstétrica é tema de audiência pública no MP-SP [Internet]. 2014 [acesso 2015 Set 1]:9-11. Disponível em: http://www. mpsp.mp.br/portal/page/portal/noticias/noticia?id_noticia $=12741378 \& i d$ grupo $=118$

24. Diniz CSG. Entre a técnica e os direitos humanos : possibilidades e limites da humanização da assistência ao parto [tese]. São Paulo (SP): Faculdade de Medicina da Universidade de São Paulo; 2001.

25. Alves $M$, Silva $A$. Avaliação da qualidade de maternidades: assistência à mulher e seu recém-nascido no SUS. São Luis: UFMA/Unicef; 2000.

26. Ratto K. Maternidade Leila Diniz (1994 a 1996): nascimento de um novo profissional de saúde? ? Rio de Janeiro: Instituto Fernandes Figueira; 1997.

27. Hotimsky SN, Aguiar JM, Venturi Junior G. A violência institucional no parto em maternidades brasileiras. In: : Venturi G, Godinho T, organizadores. Mulheres brasileiras e gênero nos espaços público e privado - uma década de mudanças da opinião pública. São Paulo: Perseu Abramo/Sesc-SP; 2013. p. 217-29.

28. Carroli G, Mignini L. Episiotomy for vaginal birth. Cochrane Database Syst Rev. 2009; (1):CD000081. doi: 10.1002/14651858.CD000081.pub2.

29. Scott JR. Episiotomy and vaginal trauma. Obstet Gynecol Clin North Am [Internet]. 2005 [acesso 2015 Set 9]; 32(2):307-21. Disponível em: http://www.ncbi.nlm.nih.gov/ pubmed/15899363

30. Assembléia Legislativa do Estado de São Paulo (ALESP). Vítimas de estupro depõem na CPI que apura violações de direitos humanos em universidade [Internet]. 2012 [acesso 2009 Maio 20]:1-24. Disponível em: http://www.al.sp.gov.br/noticia/?id=361315

31. Organização Mundial de Saúde. Prevenção e eliminação de abusos, desrespeito e maus-tratos durante o parto em instituições de saúde. Genebra: OMS; 2014. 
O ensino de obstetrícia no Brasil tradicionalmente requer que o aluno realize um certo número de procedimentos para que seja avaliado, o que levou a uma cultura de ensino do uso não informado, não consentido, das vaginas das parturientes mais pobres, usuárias do SUS, pelos alunos de medicina e outras profissões, para o treinamento de habilidades cirúrgicas. Tais abusos permanecem normalizados por uma cultura institucional que não os reconhece como violações de direitos, promovendo um ensino de habilidades descolado do ensino de valores e dos direitos das usuárias. Para promover um diálogo entre os setores envolvidos, realizamos em março de 2015 o evento "A Vagina-escola: seminário sobre violência contra a mulher no ensino das profissões de saúde". O seminário foi especialmente oportuno por acompanhar a recente declaração da Oorganização Mundial de Saúde (OMS) "Prevenção e eliminação de abusos, desrespeito e maus-tratos durante o parto em instituições de saúde", reforçando a urgência do tema na Saúde Coletiva.

Palavras-chave: Violência contra a mulher. Relações de gênero. Assistência ao parto. Ensino das profissões de saúde.

\section{The vagina-school: interdisciplinary seminar on violence against woman in the teaching of the health professions}

The teaching of obstetrics in Brazil traditionally requires the student to perform a number of procedures to be evaluated, which led to an uninformed use, not consented, the vaginas of the poorest mothers by students of medicine and other professions, for the surgical skills training. Such abuses remain standardized by an institutional culture that does not recognize them as rights violations, promoting teaching skills taken off the teaching of users values and rights. To promote a dialogue between the sectors involved, in March 2015 the event "The Vagina school: seminar on violence against women in the teaching of the health professions" was promoted. The workshop was especially opportune for following the recent World Health Organization (WHO) statement "Prevention and elimination of abuse, disrespect and ill-treatment during childbirth in health institutions", reinforcing the urgency of the theme in Public Health.

Keywords: Violence against woman. Gender relations. Childbirth care. Teaching of the health professions.

\section{La vagina-escuela: seminario interdisciplinario sobre violencia contra la mujer en la ensenanza de las profesiones de salud}

La ensenanza de partería en Brasil requiere el estudiante una serie de procedimientos para ser evaluado, lo que llevó a un uso desinformado, no consentido, de las vaginas de las madres más pobres por estudiantes de medicina y otras profesiones, para la formación de habilidades quirúrgicas. Tales abusos permanecen estandarizadas por una cultura institucional que no reconoce como violación de los derechos, la promoción de habilidades de enseñanza sacada de valores y derechos de los usuarios. Para promover el diálogo entre los sectores involucrados, fue realizado el evento "La Vagina-Escuela: seminario sobre la violencia contra la mujer en la enseñanza de los profesiones de salud." El taller fue especialmente oportuno, después de la reciente declaración de la Organización Mundial de la Salud (OMS) "Prevención y eliminación del abuso, falta de respeto y los malos tratos durante el parto en instituciones de salud", lo que refuerza la urgencia de la cuestión en la salud pública.

Palabras clave: Violencia contra la mujer. Relaciones de género. Atención al parto. Ensenanza de las profissiones de salud. 
